\title{
Determination of multiple solutions of load flow equations
}

\author{
PANKAJ MAHATA* and P S NAGENDRA RAO \\ Department of Electrical Engineering, Indian Institute of Science, Bangalore 560012, India \\ e-mail: pankaj.mahata076@gmail.com; nagendra@ee.iisc.ernet.in
}

MS received 1 August 2015; revised 27 December 2015; accepted 18 March 2016

\begin{abstract}
This paper is concerned with the problem of finding all the real solutions (all components of the solution vector must be real values) of load flow equations. Solutions in which some of the components are complex values are of no interest as they have no physical significance as a load flow solution. This problem is significant not only because of its theoretical challenge but also, its relationship with several system behavior related issues. Approaches suggested so far for solving this problem are rather ad hoc, computationally demanding and have been demonstrated only on very small systems. Further, it has been subsequently shown by others that many of these methods are not capable of finding all solutions. In this work a new approach is proposed which is more systematic and seems to have the potential to handle even large problems. We show that for any system it is possible to find the multiple load flow solutions (MLFS) corresponding to a given operating point extremely easily, starting from a set of points that are referred to as zero load solutions (ZLS) in this paper. It is shown that the complete set of ZLS is unique for a system and MLFS for any other operating point can be obtained starting from these ZLS using only the Newton's load flow method. The set of procedures for implementing the proposed scheme are illustrated and their features are highlighted by considering several sample systems.
\end{abstract}

Keywords. Load flow; multiple solutions; zero load condition; zero load solution; voltage stability; critical point; continuation; manifold.

\section{Introduction}

Load flow analysis methods are extremely important for the planning and operation of modern power systems. This is widely recognized and is also reflected in the research concerning this problem in the last six decades. Most of this work is focused towards developing more and more efficient solution methods. However, in recent years there is considerable interest in understanding the nature of the load flow equations and their solutions. The initial impetus to this type of work came from a need to answer questions such as whether a converged solution is feasible or infeasible; if it is feasible, does it imply there are multiple real solutions? etc. Korsak [1] and later Johnson [2] tried to address these questions and showed that there could be more than one real solution for a given set of load flow equations. One of the significant early work in this area is by Baillieul and Byrnes [3]. This theoretical work is based on the analysis of load flow equations of 3-node and 4-node networks with no PQ buses, zero real power injection at all nodes and considering all lines to be of equal reactance (no resistance). They showed that a 3-node network could have up to six load flow solutions. In addition, they proposed an

*For correspondence upper bound for the maximum number of solutions (both real and imaginary) which is ${ }_{2 N} C_{N}$ i.e. $\frac{2 N !}{N ! N !}$. This bound is much smaller than $2^{2 N}$-the bound given by the classical Bezout theorem.

More recently it has been shown by Sauer and Pai [4] that finding answers to these questions can also help to answer some behavior related issues such as voltage stability and voltage collapse. Tamura et al [5] have found close association between multiple load flow solutions (MLFS) and proximity to voltage collapse. These issues have also found importance in the context of assessing system stability using MLFS by Yorino et al [6].

There have also been other methods proposed by Tamura et al [7], Salam et al [8], Zhao et al [9], Ma and Thorp [10] and Zhigang et al [11] to find all the MLFS. Among these methods the method proposed by Salam et al [8] is capable of finding all MLFS. But as stated in it, this method is not useful for handling system having more than 10 nodes. Even though the method proposed by Ma and Thorp [10] appears attractive because of the reduced computational effort, it is shown by Molzahn et al [20] that the method is not capable of finding all MLFS for a given system. The other three methods by Tamura et al [7], Zhao et al [9], Zhigang et al [11] assume that the total number of MLFS cannot exceed $2^{N}$ for an $(N+1)$ bus system. But it is 
shown later in this paper that for several systems it is possible to find more than $2^{N}$ MLFS and hence, even if we succeed in finding $2^{N}$ solutions using these methods, they may not represent all the solutions.

There are other investigations that address some related problems. Iba et al [12] show that Newton-Raphson rectangular method is more suitable compared to NewtonRaphson polar method to find low voltage solutions. They also propose a method of using the optimal multiplier proposed by Iwamoto and Tamura [13] to get a second load flow solution near a critical loading point. Overbye and Klump [14] proposed an alternate approach for this problem. Liu et al [15] have proposed a method to compute all type-1 load flow solutions.

The aim of this paper is to propose a method for finding all the real (valued) solutions of the load flow equations. The proposed method is based on a very simple idea that the load flow solutions corresponding to varying magnitudes of a given load pattern are connected and constitute a manifold. On this manifold there must be necessarily a point corresponding to a zero loading condition. The basic premise of the proposed scheme is that all possible zero load solutions (ZLS) can be found very easily. Since these points also lie on the manifolds (loci of a sequence of load flow solutions obtained by multiplying the injections at all buses by a constant parameter) corresponding to a sequence of solutions, all the manifolds can be traced completely starting from these ZLS. In fact, it is shown here that this tracing can be done by using only the Newton's method.

The method proposed here is also different from earlier ones in that, the earlier set of methods essentially seek to find the MLFS for a given loading condition. But, the method proposed here enables not only to find the MLFS for a given loading condition but also at any other load condition where all the given loads at PQ buses and P at all the PV buses are multiplied by a common scalar $\lambda$. The scalar $\lambda$ defines the load level; when it equals 1.0 it corresponds to the base case load and when it is zero defines the ZLS condition. The relative values of all the node injections (other than $\mathrm{Q}$ injection at $\mathrm{PV}$ buses) remain constant as $\lambda$ is varied; this set of relative values define a load profile (pattern) and $\lambda$ the load level. In addition, the MLFS for any other loading profile of the system could also be found starting from the unique ZLS.

\section{Proposed method}

The proposed method essentially involves three steps. The first step is to enumerate all the zero load conditions. The second step is to identify the set of zero load conditions that are relevant to the given load distribution (profile) and obtain the corresponding ZLS. The third is to trace a number of load flow solution trajectories starting from this set of computed ZLS. In this section first we discuss the method of enumerating all zero load conditions, then the method of finding the ZLS corresponding to a zero load condition. With these procedures we can determine all ZLS for a given system, which is unique for a system and is independent of its load profile and load level. With this set of ZLS it will be possible to find the MLFS for any loading condition. However a procedure for limiting the calculation of ZLS only to the required set corresponding to a given load is also given.

\subsection{Zero load solutions (ZLS)}

A zero load solution is the system voltage profile when the real and reactive power injection at all the PQ buses and the real power injection at all the PV buses are zero. At any PQ bus a zero load condition can be simulated in two ways; considering the load to be either an open circuit or a short circuit. This cannot be done in the case of PV buses. Hence, we consider these cases one by one.

2.1a Systems having only $P Q$ buses: For a system having N no. of PQ buses and a slack bus, it is easy to see that it is possible to have $2^{N}$ different zero load patterns. However, all of them need not result in a distinct voltage profile in the network. For example, for $(N+1)$ bus radial system with slack bus at one end, only $(N+1)$ distinct ZLS can be found. However, for some networks it is indeed possible to have $2^{N}$ distinct ZLS. An example of this type is a $(N+1)$ bus system whose network (graph) is a clique.

As an example of a typical system we consider the five bus system taken from Salam et al [8]. This system has 4PQ buses and we can choose the zero load conditions in $2^{4}$ ways. Of these 16 possible conditions only 12 distinct conditions can be realized and the other four cases will be equivalent to some of these 12 conditions. The distinct conditions under which different voltage profiles can be obtained and also the four equivalent conditions are listed in table 1 and the 12 distinct voltage profiles are given in table 2 . These conditions are obtained by inspection as the network is small.

A scheme to determine all the ZLS must essentially have two main steps:

(1) A step for enumerating the distinct zero load conditions and

(2) A step for computing the voltage profiles for each of these network conditions.

In order to find the set of distinct ZLS we have to eliminate the 'equivalent cases' from the maximum possible number of $2^{N}$ cases. The idea behind the proposed scheme to do this is as follows. Consider the ZLS No 11 in table 1, for the five-bus system shown in figure 1 . In this case loads at nodes 2 and 4 are considered as 'short circuits'. Hence the voltage of node 5 will be zero irrespective of whether node 5 is open or short circuited. Therefore short circuiting 2, 4 and 5 to ground is identical to short circuiting only 2 and 4 . 
Table 1. Zero load conditions for the five bus system.

\begin{tabular}{llc}
\hline Sl no & Zero load condition & Equivalent cases \\
\hline 1 & All open circuit & \\
2 & Short circuit at $3,4,5$ & \\
$3-6$ & Short circuit at $2 / 3 / 4 / 5$ & \\
7 & Short circuit at 3 and 4 & \\
8 & Short circuit at 3 and 5 & \\
9 & Short circuit at 2 and 5 & Short circuit at $(2,5$ and 4$)$ ' '5' gets isolated \\
10 & Short circuit at 4 and 5 & s.c at $(2,3,4)$ or s.c at $(2,3,5)$ or s.c at $(2,3,4,5)$, '4, 5' get isolated \\
11 & Short circuit at 2 and 4 & \\
12 & Short circuit at 2 and 3 &
\end{tabular}

Table 2. Zero load solutions of five bus system with only PQ buses.

\begin{tabular}{llllll}
\hline Condition no & Bus 1 & Bus 2 & Bus 3 & Bus 4 & Bus 5 \\
\hline 1 & 1.0600 & 1.0600 & 1.0600 & 1.0600 & 1.0600 \\
2 & 1.0600 & 0.4892 & 0 & 0 & 0 \\
3 & 1.0600 & 0 & 0.2695 & 0.2156 & 0.0719 \\
4 & 1.0600 & 0.6115 & 0 & 0.1223 & 0.4485 \\
5 & 1.0600 & 0.6112 & 0.1814 & 0 & 0.4075 \\
6 & 1.0600 & 0.6784 & 0.6437 & 0.5859 & 0 \\
7 & 1.0600 & 0.5782 & 0 & 0 & 0.3855 \\
8 & 1.0600 & 0.4991 & 0 & 0.0644 & 0 \\
9 & 1.0600 & 0 & 0.2561 & 0.1982 & 0 \\
10 & 1.0600 & 0.5152 & 0.1691 & 0 & 0 \\
11 & 1.0600 & 0 & 0.1026 & 0 & 0 \\
12 & 1.0600 & 0 & 0 & 0 & 0 \\
\hline
\end{tabular}

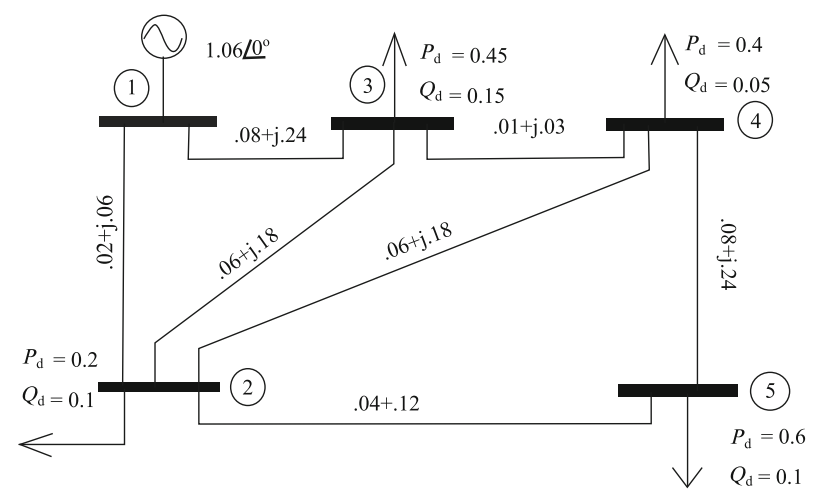

Figure 1. Five-bus network.

And these two cases are considered equivalent since the ZLS voltage profile for both the cases is same. In this case node 5 is considered as the isolated node and nodes 2 and 4 are considered as 'boundary nodes', nodes 1, 3 are considered as internal nodes. It is easy to see that 'equivalent cases' arise in situations, when a subset of buses where the load is considered as a short circuit (nodes 2 and 4 in the example) can force the voltage at another set of buses (node 5 in the example) to zero (in effect ground them). This set of nodes which are forced to zero voltage will be connected to the rest of the network only through the nodes where the load is considered as a short circuit. This set of short circuited nodes could be considered as a set of 'boundary nodes' whose removal would separate the 'isolated nodes' (the set of nodes whose voltage is forced to zero) from the rest of the network nodes whose voltage is nonzero in the ZLS (internal nodes). As an additional example consider ZLS 12 in table 1. Here nodes 2 and 3 are boundary nodes, 4 and 5 are isolated nodes and only node 1 is the internal node.

For systems considered here, the ZLS has been enumerated by inspection. However for larger systems, we need an algorithm for this purpose. Such an algorithm has been developed by Rawal [16] and it is also shown for larger systems (system with greater than 100 node) the no. of ZLS becomes quite large even though it continues to be less than $2^{N}$.

Given the list of distinct network conditions corresponding to the zero load, finding the voltage profile is straight forward. This essentially involves linear network analysis of passive networks with one known voltage source at the slack bus as shown below.

$$
\left[\begin{array}{c}
I_{\mathrm{sl}} \\
I_{\mathrm{BUS}}
\end{array}\right]=\left[Y_{\mathrm{BUS}}\right]\left[\begin{array}{c}
V_{\mathrm{sl}} \\
V_{\mathrm{BUS}}
\end{array}\right]
$$

where sl denotes slack bus

In Eq. (1) $V_{\mathrm{BUS}}$ and $I_{\mathrm{BUS}}$ correspond to only the PQ nodes which are considered to be an open circuit in the particular ZLS; correspondingly, the rows and columns of PQ nodes where a short circuit is considered as the zero load are excluded from the full $Y_{\mathrm{BUS}}$ of the network. In Eq. (1), $I_{\mathrm{BUS}}=0$ as there are no injections at any of the PQ nodes (where load is an open circuit) and $V_{\mathrm{sl}}$ is the known slack bus voltage,

Hence, the voltage at all PQ nodes (where load is an open circuit) is given by 


$$
V_{\mathrm{BUS}}=-\left[\widehat{Y}_{\mathrm{BUS}}\right]^{-1}\left[Y_{\mathrm{BUS}}^{\mathrm{sl}}\right] V_{\mathrm{sl}}
$$

where $\hat{Y}_{\mathrm{BUS}}$ is $Y_{\mathrm{BUS}}$ excluding the slack bus row and column and $Y_{\mathrm{BUS}}^{\mathrm{sl}}$ is the column of $Y_{\mathrm{BUS}}$ corresponding to slack bus (considering rows of only PQ nodes (where load is an open circuit)).

It must be noted that the computation indicated in Eq. (2) has to be carried out for each of the distinct zero load conditions. This computation itself could be quite demanding. However, we propose a scheme here to carry out this computation easily. Note that $Y_{\mathrm{BUS}}^{\mathrm{sl}}$ is an extremely sparse column. The maximum possible number of nonzero elements in this vector is equal to the number of lines (say k) incident to slack bus. Hence, we need only the corresponding k columns of $\widehat{Y}_{\mathrm{BUS}}^{-1}\left(\right.$ or $\left.Z_{\mathrm{BUS}}\right)$ for obtaining $V_{\mathrm{BUS}}$ in Eq. (2). The second feature to be noted is the following. Let $Z_{\mathrm{BUS}}$ be the impedance matrix of the network, with no buses connected to ground through short circuit. Now if one of the nodes of the network is connected to ground through a short circuit, the modified $Z_{\mathrm{BUS}}$ can be obtained very easily. Consider that a node (say p) is connected to ground. Then the modified $Z_{\mathrm{BUS}}$ can be obtained by using Eq. (3) given in "Computer-Aided Power System" Analysis by Kusic [17].

$$
Z_{i j}^{\text {mod }}=Z_{i j}-\frac{Z_{p i} Z_{j p}}{Z_{p p}} .
$$

This modifies all the elements of $Z_{\mathrm{BUS}}$ and the $p^{\text {th }}$ row and column to zero. So we can ignore the original $p^{\text {th }}$ row and column. This procedure can be repeatedly used if more than one node is connected to ground. The modification can be limited to only the $\mathrm{k}$ columns of the $Z_{\mathrm{BUS}}$ corresponding to the location of the nonzero elements of $Y_{\mathrm{BUS}}^{\mathrm{sl}}$. Hence, finding the voltages at all nodes corresponding to all the zero load condition is quite simple and can be carried out efficiently.

2.1b Systems with only PV buses: A zero load condition at a PV bus is defined as the condition where the real power injection at that bus is zero and the voltage magnitude of the bus is at the specified value. In systems having PV buses when we consider real power injections at all PV buses to be zero, the Q output of PV buses could be nonzero (and will be unknown) depending on the specified voltage magnitudes at other PV buses. The zero load condition at a PV bus cannot be simulated by having an open circuit or a short circuit at that bus (as could be done in the case of PQ buses). Equations of the system representing this condition at PV buses will be essentially the load flow equations. Hence, the voltage profiles corresponding to zero load condition of these systems cannot be obtained by linear circuit analysis; in fact, this has to be obtained by solving the load flow equations representing this condition. Note that the Q limits at PV buses are not considered here.
The number of MLFS, possible for a system with only PV buses, is not known. A bound of $\left(\begin{array}{c}2 N \\ N\end{array}\right)$ is proposed by Baillieul and Byrnes [3] for any system where $N$ is the number of buses excluding the slack bus. However, this bound is actually for all the solutions. It includes both real and complex solutions. Hence, we resort to a search procedure to enumerate all possible real solutions. Since, the voltage magnitudes of all the PV buses are known only their angles are unknown. Since, the angle could take any value between $0^{\circ}$ and $360^{\circ}$, we choose four different angles as the initial guess for each bus so as to cover each of the four quadrants. Generally, we use $45^{\circ}, 135^{\circ},-45^{\circ},-135^{\circ}$ for each of the buses. With $m$ number of PV buses we get $4^{m}$ choices for the initial guess solution, requiring $4^{m}$ load flow solutions. Many of them may converge to same solutions and in quite a few cases the iterations may diverge. The set of all distinct (converged) solutions is taken as the set of ZLS for the system. The choice of four angles at $90^{\circ}$ separation has been arrived at by considering several test cases and a number of alternate choices for initial guess solutions.

2.1c Systems with both $P Q$ and $P V$ buses: This type of systems are more representative of practical systems. In order to find all the ZLS for these systems a procedure is evolved here, combining the ideas presented in the two previous subsections. It is easy to see that for these systems under the zero load conditions, if the complex voltages at PV buses are known, the voltages at all the PQ buses can be calculated because there are no other sources or injections connected at the PQ buses. From Section 2.1a it is clear that the zero load condition can be simulated in a number of different ways when we consider only PQ buses. Hence, the proposed procedure is based on the following approach.

Considering only the PQ buses, we enumerate all possible distinct network conditions. For each of these conditions, the resulting system is reduced to a system of only PV buses by eliminating all the PQ buses in the network. The resulting reduced system would contain only the PV buses and the slack bus. The number of ZLS for each of these reduced systems are found by the search procedure indicated in Section 2.1b. The total number of ZLS would be the sum of all such solutions obtained for each of the reduced systems. The proposed step by step procedure is as follows:

(i) Enumerate all the distinct zero load conditions for only the PQ buses using the procedure in Section 2.1a, notionally considering all the PV buses merged with the slack bus in the modified system (this modified system will be a system with only PQ buses where all the lines connected to PV buses in the original system, will be connected directly to the slack bus). 
(ii) For each of the distinct zero load conditions found in step(i) determine the $Y_{\text {BUS }}$ matrix and arrange it as follows:

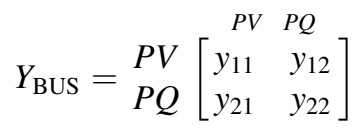

It may be noted that the set of PQ buses in Eq. (4) does not include PQ buses that are either grounded or forced to zero voltage (i.e. boundary nodes and isolated nodes). The admittance of lines incident to boundary nodes from internal nodes are however included in the corresponding diagonal terms as connections to ground. The lines incident to the isolated nodes will be absent from the $Y_{\mathrm{BUS}}$ in Eq. (4). The set of PV buses in Eq. (4) includes the slack bus also.

(iii) Find the $Y_{\mathrm{BUS}}^{\text {reduced }}$ as

$$
Y_{\mathrm{BUS}}^{\text {reduced }}=y_{11}-y_{12} \times y_{22}^{-1} \times y_{21} \text {. }
$$

(iv) Find the number of distinct ZLS for this reduced system with only PV buses and the network represented by $Y_{\mathrm{BUS}}^{\text {reduced }}$ in Eq. (5), as indicated in Section 2.1b.

(v) The voltages at the PQ buses in Eq. (4) can be now obtained by using the linear network analysis approach as explained in Section 2.1a. The only difference being, in this case in addition to the slack bus voltage, other PV buses voltages are also known.

Hence, the total number of ZLS would be the sum of the number of converged load flow solutions in step (iv) for each of the distinct zero load conditions for the PQ buses found in step (i).

2.1d Identifying ZLS that correspond to a given load: In this section 2 , so far the method of finding all the distinct ZLS has been described. This is necessary when we are interested in finding MLFS for any type of load distribution (load profile) in the system. If the interest is in finding MLFS for a given load, it is possible to reduce the number of ZLS calculations as follows.

As mentioned in earlier in section 2.1a the first step while computing ZLS is enumerating all the distinct zero load conditions. When once a zero load condition is identified all the PQ nodes in the system can be grouped into three sets, I (internal), B (boundary) and IS (isolated). For some cases one or more of these sets could be null sets. Considering the given load condition we can determine the set L, of PQ nodes where the specified load is non-zero. For the given load, a particular ZLS will be relevant only if $\mathrm{L}$ is a subset of I (internal loads). It is easy to see the logic behind this principle. For tracing the trajectories, we start with the ZLS, increment $\lambda$ from zero by a small value and multiply the given injection at each of the nodes by $\lambda$, to choose the load condition corresponding to the first point on the solution locus. If for the given load, the particular zero load condition under consideration is such that some of the boundary/isolated nodes will have to support a non-zero load, then such a condition is not feasible. Hence, such a zero load condition need not be considered for finding the MLFS for the given load and therefore the corresponding ZLS need not be calculated.

As an example consider the system given in figure 1. For the loads given in figure 1, the set of nodes with non-zero load is $\mathrm{L}=(2,3,4,5)$. For the zero load condition 11 in table 1 , that is obtained when the zero loads at nodes 2 and 4 are considered to be short circuits, the sets of internal, boundary and isolated nodes are respectively $\mathrm{I}=(3), \mathrm{B}=(2$, 4) and IS $=(5)$. Here, we see that $L$ is not a subset of I and therefore, if we are interested in the MLFS for the given load only, the ZLS corresponding to this condition 11 need not be used and hence, it need not be calculated. For the given load condition, a similar logic holds good for ZLS no 12 also, in table 1.

\section{Tracing the solution loci}

This procedure is needed to find the solutions at various loads. The basic idea behind the algorithm is to exploit the connectedness of a sequence of solutions corresponding to a monotonically changing magnitude of a load pattern (profile). This tracing can also be done by using existing techniques such as the continuation load flow by Ajjarapu and Christy [18] or the less known trajectory following procedure suggested by Price [19]. However, we propose a much simpler scheme that involves only repeated use of the Newton's load flow method in Cartesian coordinates.

In the procedure proposed here the process of tracing a solution trajectory for any given load profile involves, starting at one of the appropriate ZLS. This point corresponds to the solution of the load flow equations with all the loads in the given profile multiplied by a value of $\lambda=0$ (where $\lambda$ is a multiplier to the $\mathrm{P}, \mathrm{Q}$ load and $\mathrm{P}$ generation). A value of $\lambda=1$ for this multiplier corresponds to the given load profile. Starting with the $\lambda=0$ point we find the next point on the trajectory by incrementing $\lambda$ by a value of $\Delta \lambda$. A simple NR load flow algorithm is used to calculate the solution.

Tracing the complete trajectory requires increasing $\lambda$ up to a maximum possible value $\left(\lambda_{\max }\right)$ and then reducing it to reach $\lambda=0$. The new $\lambda=0$ point will be another ZLS different from the starting point. The process is continued further by reducing $\lambda$ till we reach $\lambda_{\min }$. From this point repeat the above procedure of increasing and decreasing $\lambda$ in small steps, till we reach the starting point (ZLS). Tracing the complete load flow solution trajectory is useful as it facilitates: 
(i) finding the multiple load flow solutions at different loading levels,

(ii) determining the loadability limits, and

(iii) finding one or more additional ZLS (if all of them have not been determined already).

\subsection{Crossing critical points}

When we get to the proximity of points when $\lambda$ is (locally) a maximum or minimum, the simple NR method used for tracing the curve could fail to converge. This is because at such critical points the load flow jacobian becomes singular. Hence, we need a slightly different procedure to cross such points and complete the tracing. These critical points correspond to the steady state stability limits for the load profile being considered. There are methods in the literature essentially to find pairs of solutions close to the steady state stability limits other than continuation [18], curve tracing methods [19]. Iba et al [12] and Overbye and Klump [14] proposed algorithms for handling this problem. We propose a simple algorithm here for this purpose which enables finding pairs of solutions in the neighborhood of critical points using only the NR method. In order to develop this scheme, we use an intermediate result from Overbye and Klump [14]. Consider figure 2 which shows a part of a solution trace (one of the components of the solution plotted w.r.t $\lambda$ ). Following the notation used by Overbye and Klump [14], the load flow equations can be represented by

$$
S=F(V)
$$

where $S$ is the vector of specified quantities (loads) and $V$ is the vector of real and imaginary part of the voltages. Let $V^{m}$ be the critical point. While tracing the manifold let $V_{1}$ be the closest point to $V^{m}$ for which a successful load flow has been obtained using the Newton's load flow. Let $V^{s}$ be the point closest to $V_{1}$ for which the load flow has been obtained while reaching $V_{1}$.

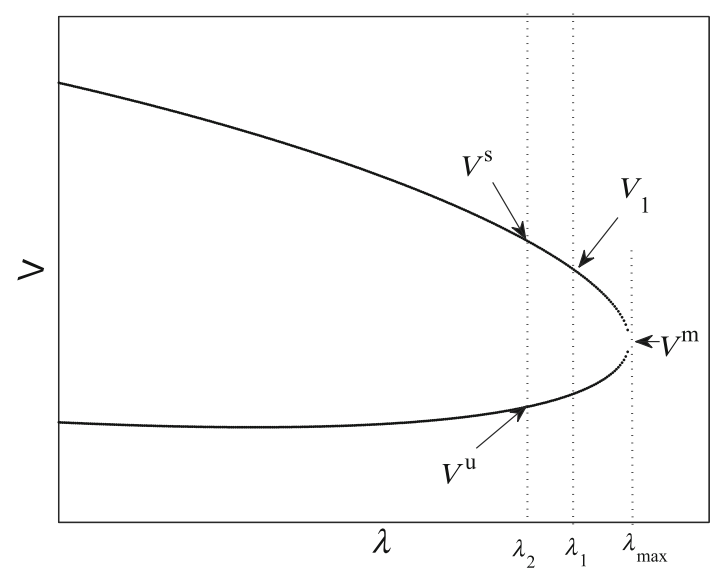

Figure 2. A part of a manifold near a critical point.
It is shown by Overbye and Klump that under such conditions

$$
\begin{gathered}
2\left[J\left(V^{s}\right)\right]^{-1}\left[F\left(V^{m}\right)-F\left(V^{s}\right)\right]=\Delta V^{m} \\
\text { where } \Delta V^{m}=V^{s}-V^{m}=V^{m}-V^{u} .
\end{gathered}
$$

The point $V^{u}$ is such that $F\left(V^{s}\right)=F\left(V^{u}\right)$.

In the method proposed here, for finding $V^{u}$, with $V^{s}$ and $V_{1}$ known, we consider $V_{1} \simeq V^{m}$ (because the NR method can successfully reach points very close to $V^{m}$ ) and using Eq. (8), we see that

$$
V^{s}-V^{u} \simeq 2 \Delta V^{m}
$$

where $\Delta V^{m}$ is computed using Eq. (7) considering $V^{m} \simeq V_{1}$.

Because of this approximation, $V^{u}$ is found using $\left(V^{s}-2 \Delta V^{m}\right)$ as an initial guess solution by the Newton's method since we know that $F\left(V^{u}\right)=F\left(V^{s}\right)$.

The proposed procedure for crossing a critical point can be summarized as follows:

1. At the beginning of the tracing, starting at $\lambda=0$, an appropriately chosen for the increment $\Delta \lambda$ would have been made. After some steps the solution tends to diverge when a critical point is close by. Then smaller increments of $\Delta \lambda$ are used to continue tracing. This process is continued till Newton-Raphson method diverges with the lowest possible value for $\Delta \lambda$ (say of the order of $10^{-4}$ ).

2. Find the change in voltage vectors $\Delta V$ between the last (corresponding to $V_{1}$ in figure 2) and its immediate previous (corresponding to $V^{s}$ in figure 2) solution as $\Delta V=\left(V^{s}-V_{1}\right)$. Considering the system load as equal to $F\left(V^{s}\right)$, obtain another load flow solution by using an initial guess of $\left(V^{s}-2 \Delta V\right)$. This solution converges to a point $V^{u}$ on the other side of the critical point $V^{m}$.

\subsection{Procedure for tracing the trajectory of solutions}

1. Start with one of the ZLS $(\lambda=0)$ found above as the starting point for tracing. Increase the value of $\lambda$ by a small amount and find the load flow solution using NR method in rectangular coordinates.

2. Continue with the above procedure every time using the solution found for the previous $\lambda$ value as the initial guess for the next $\lambda$ till we reach a point where the NR method tends to fail to provide a solution indicating the proximity of a critical point.

3. In order to continue tracking we need to find another point on the other side of the critical point and start decreasing $\lambda$ in small steps. This is done using the procedure in section 3.1 . 
4. The $\lambda$ value from this point is monotonically reduced to continue tracing the solution locus.

5. When we reach a value $\lambda=0$, this solution again correspond to one of the ZLS that have been found and can be flagged off as a used condition.

6. While tracing some trajectories one can encounter several critical points in the locus and the procedure in section 3.1 is followed to move across such critical points.

7. The above procedure is continued till we reach the starting point of the locus.

8. Tracing loci is continued with those ZLS which are not flagged off as used or not considered so far.

Even though it appears that tracing can be terminated whenever $\lambda=1$ corresponding to the specified load is reached or a critical point $\lambda_{\max }$ is reached even before reaching $\lambda=1$, it is necessary to obtain the complete trajectory (at least till we reach $\lambda=0$ again). This is because in situations where a trajectory has more than two critical points, it is possible that we reach one of the $\lambda_{\max }$ points, even before reaching $\lambda=1$ and subsequently it may reach $\lambda=1$. For example, see the trajectory in figure $6(\mathrm{~b})$. It must however be noted that continuing tracing the loci for negative values of $\lambda$ also is a possibility and it also has some attractive features. In large systems after finding one ZLS, if we completely trace a solution locus till we reach the starting point, we will be covering several ZLS. For example in the 14 bus system, it is seen that the manifolds plotted in figure 7(a) and 7(b) cross seven more ZLS before reaching the starting ZLS. Using this feature, it is possible to reduce the effort in computing these additional ZLS. In addition, there appears to be an inherent connection between the ZLS forming such sets. Such sets cannot be identified if we restrict ourselves to plotting the loci only for the positive values of $\lambda$. This is because, in the example mentioned earlier, the continuous plots in figure 7 (a) and 7(b) get plotted as four separate segments, corresponding to only positive values of $\lambda$. Further investigations are necessary to infer which of these two options is preferable in a given situation.

\subsection{Choosing the starting points}

While determining the multiple solutions corresponding to a given loading condition it is neither necessary to find all the zero load solutions corresponding to distinct zero load conditions nor trace the solutions starting from all of them. Such an effort would be needed only when one has to determine the maximum number of load flow solutions possible for a given system for any loading pattern. The approach used to identify the required set of ZLS for a given loading condition has been given in section 2.1d. ZLS can be associated with a set of nodes (I which are basically internal nodes) by excluding the isolated nodes and the boundary nodes from the network corresponding to the zero load condition defining the particular ZLS. For example, for the five-bus system (table 1), the set I for the zero load conditions 1 to 10 consists of all the nodes whereas for condition $11, I=(1,3)$ and for condition $12, I=(1)$. From the specified load for which the MLFS are required, the set of nodes L, where the load is nonzero can be determined. The method of identifying the relevant ZLS for this load has been explained in section $2.1 \mathrm{~d}$ and only the trajectories starting from these ZLS have to be traced. For tracing the trajectories we use the full network when we start from ZLS that correspond to the case when there are no isolated nodes (i.e. the set IS= (null)). However if there are some isolated nodes then we consider a smaller network for tracing the trajectories. Such a reduced network does not contain any of the isolated nodes and the lines that connect the isolated nodes to the boundary nodes (Further the boundary nodes are shorted to ground). As an example consider the five-bus system in figure 1. For tracing trajectories stating from ZLS 1 to 10 we use the full network. When starting with ZLS 11, we use a reduced network, retaining only nodes 1 and 3 , shorting nodes 2 and 4 to ground, there by isolating node 5 and excluding lines $4-5$ and 2-5. The ZLS 12 corresponds to the trivial case of a network with only the slack node with its neighboring nodes 2 and 3 shorted to ground; we do not start tracing any trajectory from here, but, trajectories could terminate here.

\section{Simulation results}

Results of a number of simulations are provided for illustration of the proposed method and also demonstrate the effectiveness. These results are presented for systems with only PQ buses, systems with only PV buses and systems with both PQ and PV buses separately.

\subsection{Systems with only $P Q$ buses}

As examples of this class of systems, we consider the fivebus system considered in section 2.1a and a seven-bus system from Salam et al [8]. The one line diagram of the five-bus system is given in figure 1 and the 12 zero load conditions are listed in table 1 and the corresponding ZLS are given in table 2 .

In order to find the multiple solutions for the base case load condition, we do not have to consider the ZLS 11 and 12 as there are non-zero loads at all the PQ buses. Hence, we consider only the first 10 ZLS. Starting with the zero load condition 1 (all open circuit loads), we trace manifold 1. This manifold has two critical points corresponding to $\lambda_{\max }=2.8$ and $\lambda_{\min }=-10.5$ (such critical points correspond to the voltage collapse points of the system) and it crosses the $\lambda=0$ axis corresponding to ZLS 6 . Similarly 


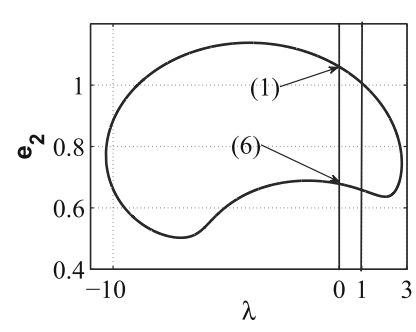

(a)

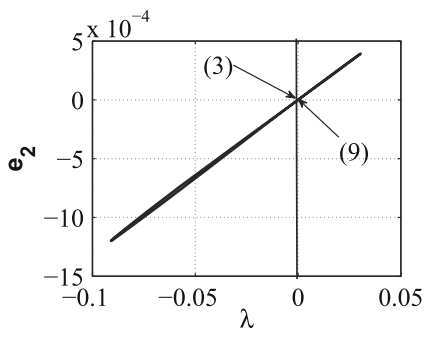

(b)

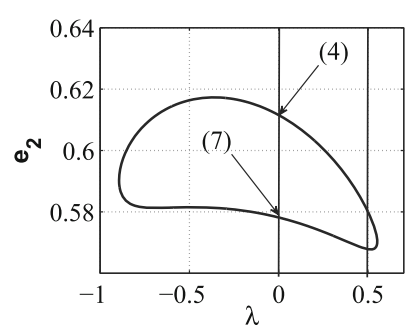

(c)

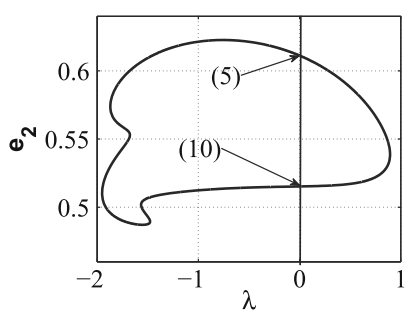

(d)

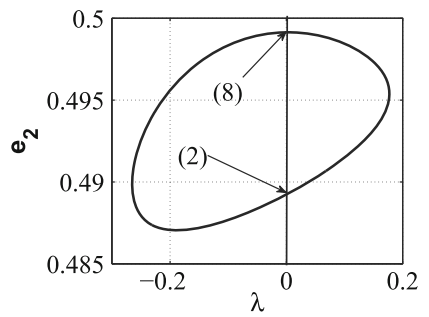

(e)

Figure 3. All manifolds $\left(e_{2}\right.$ vs $\lambda$ ) of five bus system (base case load), (a) manifold 1 (ZLS 1,6), (b) manifold 2 (ZLS 3,9), (c) manifold 3 (ZLS 4,7), (d) manifold 4 (ZLS 5,10), and (e) manifold 5 (ZLS 2,8).

we trace four more trajectories starting from ZLS 2, 3, 4 and 5. These manifolds cross the $\lambda=0$ axis at ZLS 8, 9, 7 and 10 respectively. Each of these manifolds are plotted using different scales for $\lambda$ in figure 3(a) to figure 3(e). In all these plots only the variation of $e_{2}$ w.r.t $\lambda$ is shown; $e_{2}$ is the real part of the voltage of bus no 2 . The ZLS corresponding to each of the manifolds (intersection with the $\lambda=0$, vertical line) are indicated on these manifolds in figure 3 (In all plots similar to figure 3, there are vertical lines corresponding to some significant values of $\lambda$ such as 0 and 1 to facilitate easy recognition of ZLS and MLFS). In figure 3 , the number of intersections of the $e_{2}$ plots with the vertical line corresponding to the chosen $\lambda$ value (considering all the manifolds) determines the total number of multiple solutions for that load and also the $e_{2}$ values of the corresponding load flow solutions.

It is easy to see that for $\lambda=1$ the system has two solutions located on manifold 1 (figure 3(a)). These two solutions have been obtained by other investigators also. However, the procedure here enables us to get multiple solutions for any level of loading for this load profile. For example, with $\lambda=0.5$, we get six solutions ( 2 each in manifolds 1,3 and 4). Similarly with $\lambda=0.1$, we get eight solutions (two each in manifolds 1, 3, 4 and 5) and with $\lambda=0.01$ we get 10 multiple solutions; two solutions in each of the five manifolds.

It is pointed out that from figure 3 we can read only the real part of the voltage at bus 2 , of the load flow solution corresponding to a chosen value of $\lambda$ and in order to represent the complete solution for the five bus system seven more plots similar to figure 3 corresponding to the variation of the imaginary parts of voltage at the four buses and three more plots of the real part of the voltage of the other three buses are needed (They are computed but not shown in the paper as they could seem to be repetitive). Note that the slack bus voltage remains constant throughout. If we are interested in the other components of the load flow solution vectors, we have to use the other seven sets of plots mentioned earlier (they has been computed but not plotted in the paper) to find the value of the plotted component at the intersection of the chosen $\lambda$ line and the manifold.

Another feature to note is in figure 3(d) where, it can be seen that for negative values of $\lambda$ the manifold (No. 4), has five critical points. The proposed algorithm does not encounter any difficulty in tracing such manifolds.

In order to highlight how the load profile affects the solution trajectories, we consider another load profile $[2+j 0,0,0,0]^{t}-$ a nonzero load at bus 2 and zero loads at other buses for this system. Using ZLS 1 to 10, eight solution trajectories are obtained and they are plotted (component $e_{2}$ ) w.r.t. $\lambda$ in figure 4.

Here, there are only 12 ZLS but 16 MLFS corresponding to all values of $\lambda$ in the range of -2.98 to +1.58 .

The second example that is considered is the seven bus system. This system has 30 ZLS. However, corresponding to the base case load profile only 20 of these solutions are of interest. While tracing the solution trajectories, it is seen that all these 20 ZLS are covered by tracing only six manifolds. It is seen four of these manifolds have four critical points each and the proposed scheme encounters no difficulties, while crossing them. These six manifolds (one of the component $e_{2} \mathrm{Vs} \lambda$ ) are given in figure 5(a) to figure 5(f). It is interesting to note that in the paper by Salam et al [8], 924 homotopy curves are traced for locating the four solutions corresponding to the base case load. In the approach proposed here all of these are located on only one 


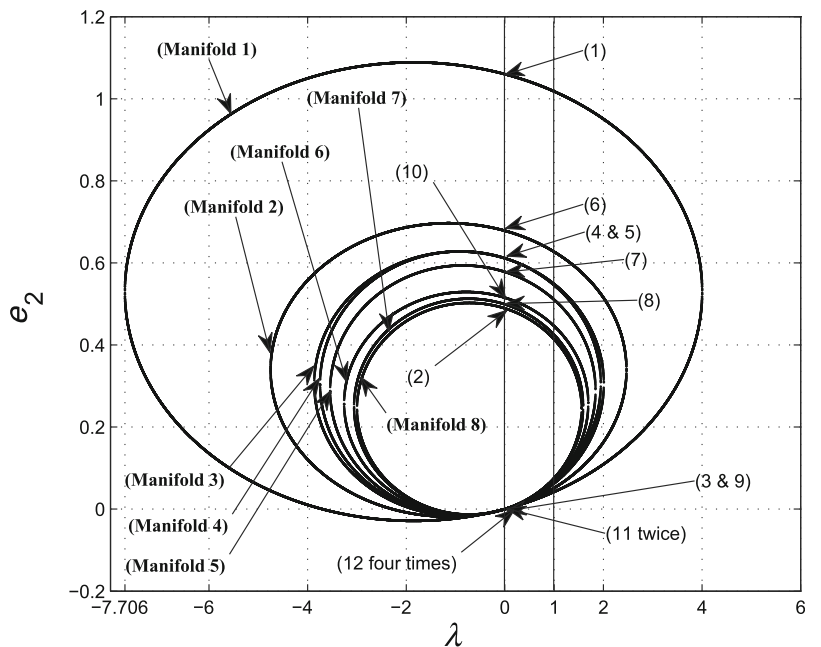

Figure 4. All ZLS and manifolds (only bus 2 loaded) of five bus system.

manifold (manifold 1, figure 5(a)). By tracing all the six manifolds, we can get the multiple solutions for any loading level of this profile. For example for $\lambda$ values of 1.0, 0.5, $0.2,0.1,0.05,0.01$ we can see from figure 5 , there are 4,4 , $4,8,10,16$ solutions respectively. It is also obvious from figure 5 , that for $\lambda$ values less than $2 \times 10^{-3}$ we can get 20 MLFS for this system.

\subsection{Systems with only PV buses}

For this class of systems, enumerating all ZLS, as done for systems with only PQ buses, does not seem possible. Even though a bound for the number of possible solutions is available, this bound is not very tight. Further, finding all the ZLS for systems with only PV buses is also important while finding the total number of multiple solutions for systems with PV and PQ buses. The clique topology is of special interest because when we reduce such systems by eliminating all PQ buses, the resulting reduced topology will almost always be a clique network.

Hence, we first consider a number of systems with clique and ring topologies of varying sizes. The data is generated considering a line impedance $(0.01+\mathrm{j} 1.0)$ p.u and adding a random value between 0 and $10 \%$ the line impedance value to each of the lines to eliminate symmetry. All the PV bus voltages are considered to be 1.0 p.u and equal to the slack bus voltage. The number of ZLS obtained are given in table 3. In table 3 , the number of ZLS for each of the systems considered is given for two more values of line resistance values, a higher value of $\mathrm{R}$ around $1.0 \mathrm{pu}$ and a lower value of $\mathrm{R}$ of about $0.0001 \mathrm{pu}$. The results in table 3 are obtained using the procedure $\left(4^{N}\right.$ trials) indicated in section $2.1 \mathrm{~b}$. However, it has been verified that it is not possible to find any higher number of solutions for any of these cases by trying out $6^{N}$ and $8^{N}$ trials for a $(N+1)$ bus system.

The only result available in the literature for this class of systems is given by Baillieul and Byrnes [3] where the number of ZLS for a three bus system with equal line admittances and no resistance is given as six. For the three bus system the results here match that of the system given by Baillieul and Byrnes and it is the only case where the number of real solutions is actually equal to the proposed bound $\left(\begin{array}{c}2 N \\ N\end{array}\right)$. For a similar symmetrical four-bus systems it is indicated that it can have 14 real solutions. The other

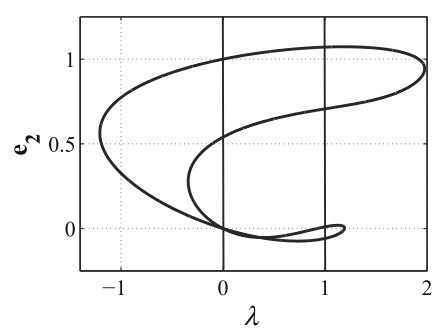

(a)

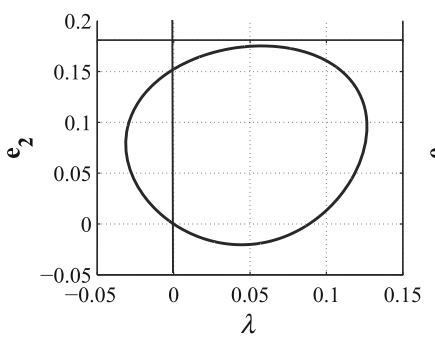

(d)

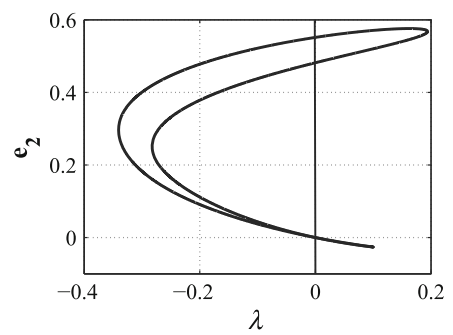

(b)

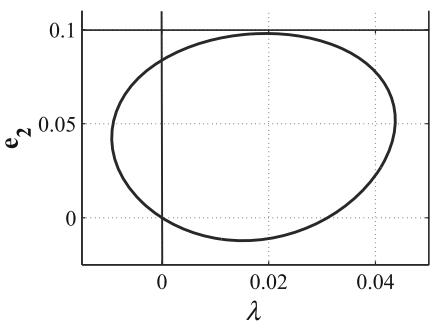

(e)

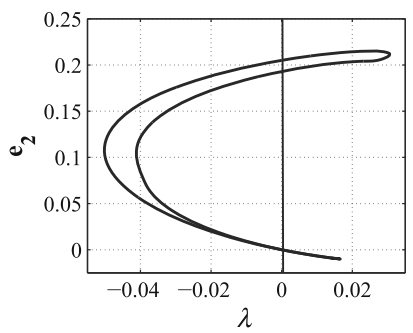

(c)

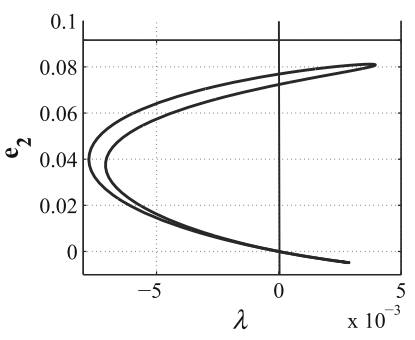

(f)

Figure 5. Six manifolds (e2 vs $\lambda$ ) of seven bus system. (a) Manifold 1, (b) Manifold 2, (c) Manifold 3, (d) Manifold 4, (e) Manifold 5, and (f) Manifold 6. 
Table 3. Number of ZLS for systems with only PV buses.

\begin{tabular}{|c|c|c|c|c|c|c|c|c|}
\hline \multirow[b]{3}{*}{ No } & \multirow[b]{3}{*}{ Size $(N+1)$} & \multicolumn{6}{|c|}{ Number of zero load solutions } & \multirow[b]{3}{*}{$\frac{2 N}{N}$} \\
\hline & & \multicolumn{3}{|c|}{ Clique } & \multicolumn{3}{|c|}{ Ring } & \\
\hline & & $R<10^{-4}$ & $R \simeq 0.01$ & $R \simeq 1$ & $R<10^{-4}$ & $R \simeq 0.01$ & $R \simeq 1$ & \\
\hline 1 & 3 & 6 & 6 & 2 & 6 & 6 & 2 & 6 \\
\hline 2 & 4 & 12 & 8 & 2 & 12 & 8 & 2 & 20 \\
\hline 3 & 5 & 30 & 22 & 2 & 30 & 30 & 4 & 70 \\
\hline 4 & 6 & 62 & 32 & 2 & 60 & 60 & 4 & 252 \\
\hline 5 & 7 & 144 & 84 & 2 & 140 & 122 & 4 & 924 \\
\hline 6 & 8 & 340 & 128 & 2 & 204 & 166 & 4 & 3432 \\
\hline
\end{tabular}

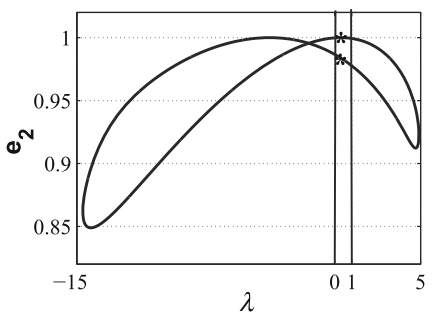

(a)

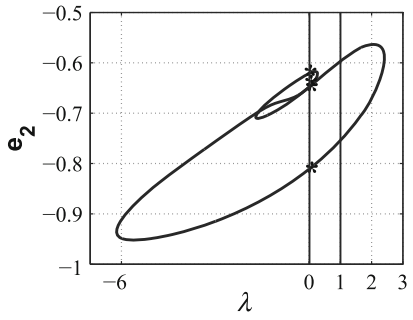

(b)

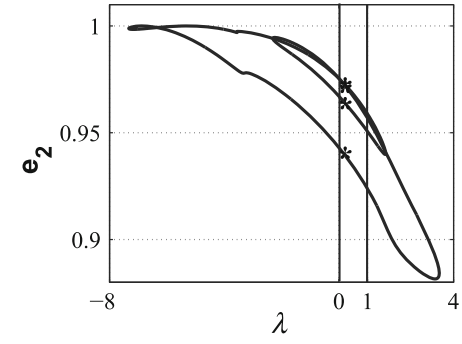

(c)

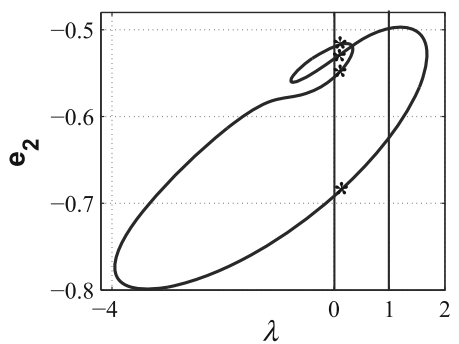

(d)

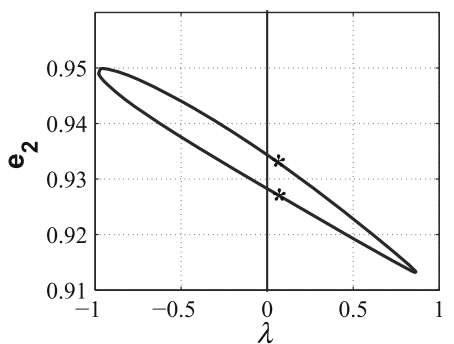

(e)

Figure 6. Manifolds ( $e_{2}$ vs $\lambda$ ) for 5 bus system. (a) Manifold 1, (b) Manifold 2, (c) Manifold 3, (d) Manifold 4, and (e) Manifold 5.

solutions (six) would be complex. From table 3 the following observations can be made

(i) For a given topology the number of ZLS is dependent on the line parameter values.

(ii) It is seen that increase in line resistances results in the decrease of the number of ZLS.

(iii) The actual number of real ZLS would be generally very small as compared with the bound of $\left(\begin{array}{c}2 N \\ N\end{array}\right)$ for systems with $N>5$.

(iv) The number of ZLS can be greater than $2^{N}$. This is evident from the result for the clique topology with very low line resistance $\left(R<10^{-4}\right)$.

Another five bus system with all PV buses considered for investigation here is taken from Molzahn et al [20]. This example has been used by Molzahn et al to show that the method by Ma and Thorp cannot work for all systems. This has been shown by giving the 10 solutions for this system whereas the method by $\mathrm{Ma}$ and Thorp succeeds in finding only two of them. The 10 solutions have been found using the method by Salam et al that requires tracing $\left(\begin{array}{l}8 \\ 4\end{array}\right)$ solutions trajectories (i.e. 70). For this system, using the proposed method in section $2.1 \mathrm{~b}$, we are able to find 16 ZLS. Starting from these ZLS, we can trace eight solution trajectories. Using these trajectories 10 solutions given by Molzahn et al are obtained. It is also seen that at reduced loads for $\lambda<0.5$, the system has 16 MLFS.

\subsection{Systems with both $P Q$ and $P V$ buses}

The proposed algorithms for this class of systems are demonstrated by considering the five bus data taken from Salam et al and the 14 bus data taken from Ph.D thesis of 
Rajathy [21]. This five bus system has three PQ buses and one PV bus. Considering the two alternatives of zero load conditions for the three PQ buses we get eight zero load conditions for PQ buses. Reducing the system in each case to a two-bus system with one PV and one slack bus, we get two real solutions for each of the eight conditions (all are distinct). Hence, totally we get 16 ZLS which are shown in the figure 6(a) to figure 6(e). Using these ZLS, five trajectories can be obtained covering all of them. These are given in figure 6. It can be seen that three of the manifolds have four critical points each and also cross four ZLS points. The other two manifolds have two critical points each and cross two ZLS. The 10 solutions for the base case have been obtained by using the trajectories shown in figure 6 . These are obtained from the first four manifolds. In addition we have been able to get 16 solutions for a reduced loading condition $(\lambda=0.1)$. These are also pointed out as " $\star$ " in the figure. It must be pointed out that this set of 10 solution for the base case has also been obtained by Salam et al. This is obtained by first tracing $2^{8}(256)$ homotopy trajectories. They subsequently show it can also be done by tracing only 70 homotopy curves corresponding to $\left(\begin{array}{l}8 \\ 4\end{array}\right)$. The advantage of the proposed scheme is obvious. With this scheme it is not only possible to get the same set of solutions with only five solution traces, but it is possible to get all the MLFS for any value of $\lambda$ for this load profile. For example it is easy to see from figure 6 that for small values of $\lambda$ (less than 0.1 ), there are 16 solutions and no solutions are possible for values of $\lambda>5$.

The last example presented is the 14 bus system. It is explicitly mentioned by Salam et al that their method cannot be even considered for finding the multiple solutions for this system because of the extremely large number of homotopy traces required. This system has $11 \mathrm{PQ}$ buses and two PV buses. In this case considering only the 11 PQ buses it is possible to enumerate 332 distinct zero load conditions (152 cases with the full network and 180 cases smaller networks). For each of these distinct conditions the system is reduced to a three bus system. Using the search method in section $2.1 \mathrm{~b}$, it is possible to find only 4 ZLS for each of these cases. And hence there are 1328 distinct ZLS for this system. Two manifolds obtained by starting from two of these ZLS, are given in figure 7. Four solution corresponding to the base case loading can be obtained from these two trajectories. For a low level of loading say $\lambda=0.2$, it is easy to see that there are 16 solutions from only these two manifolds. It is interesting to note that each of these trajectories contain eight ZLS. A noteworthy point in figure 7 , is that, we see that there are 16 critical points in manifold 1 (figure 7(a)) and eight critical points in manifold 2 (figure 7(b)). The proposed tracing scheme using only the Newton-Raphson method has no difficulty in crossing all these points while generating the complex contour.

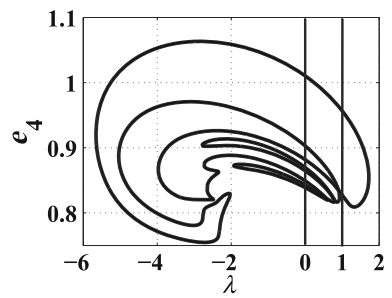

(a)

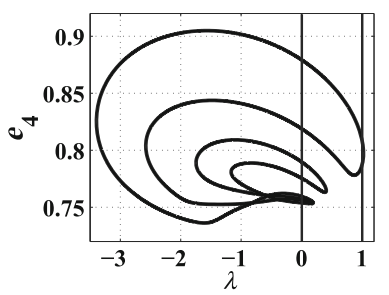

(b)
Figure 7. Manifolds for 14 bus system. (a) $e_{4}$ vs $\lambda$ (manifold 1) and (b) $e_{4}$ vs $\lambda$ (manifold 2).

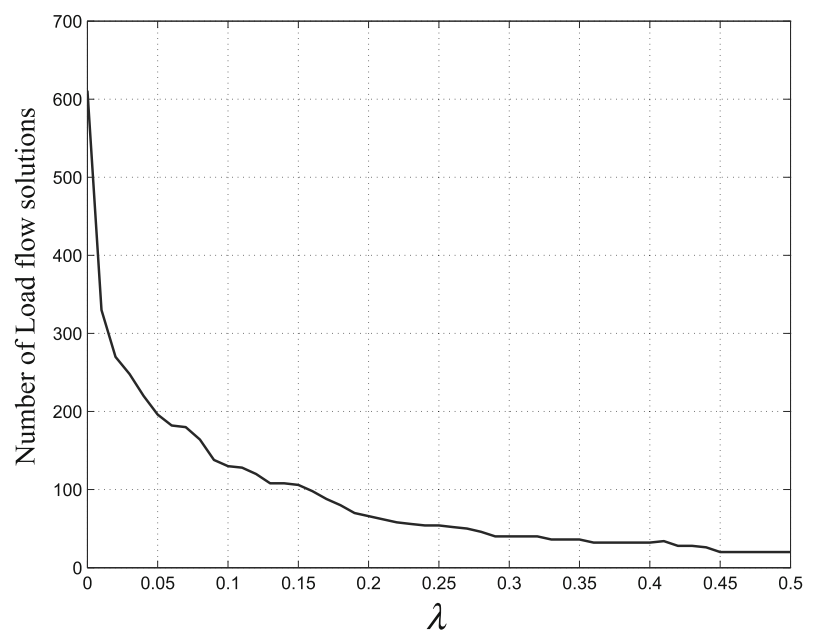

Figure 8. MLFS vs $\lambda$ (Base case load profile) for 14 bus system.

For this system considering the base case load profile, all the manifolds have also been traced only for positive values of $\lambda$. When manifolds are not traced completely (including the negative values of $\lambda$ ), each manifold is traced as a set of segments i.e manifold 1 and 2 are obtained as four separate traces each. In this case 304 such segments covering all the ZLS corresponding to the base case load profile are obtained. Based on these solution traces, the number of MLFS for different values of $\lambda$ is plotted in figure 8 (for a range of $0<\lambda<0.5$ ). For $\lambda<0.01$, we get 608 MLFS and from figure 8 , it is seen for $\lambda=0.5$ there are 20 solutions. It is seen that for $\lambda=1$, the number of MLFS is only 4 (those obtained from manifolds 1 and 2 in figure 7). The number of MLFS reduces to 2 for $\lambda>1.03$, and the critical loading corresponds to $\lambda=1.74$. Based on the investigation here, some important general observations can be made:

(1) The number of multiple solutions for a given system not only depends on the load level but also on the load profile.

(2) The presence of PV buses can increase the number of multiple solutions. This can be seen comparing two systems with identical line parameters and loads, one of them having PV buses and the other one without any. In 
general presence of PV buses seems to contribute to larger number of multiple solutions.

(3) It is generally believed that the number of multiple solutions for a given load profile reduces with increase in loading level (increasing $\lambda$ ). From this point of view a system must have the maximum number of multiple solutions at zero loading. Since, the zero load condition is same for all loading profiles, it could imply that for any type of loading, a system cannot have more number of load flow solutions than the number of ZLS. But this is not always true. Cases are reported here where the number of solutions at zero load $(\lambda=0)$ is less than at a non-zero load $(\lambda>0)$. The results here provide the answer for this phenomenon. The number of MLFS could exceed the number of ZLS, if some of the manifolds touch one another at some ZLS, as seen in figure 4.

(4) The number of ZLS is dependent only on the network topology in the case of systems with no PV buses. However, in systems having both type of buses this depends both on the topology and parameter values of the network.

(5) The number of ZLS of a network is indicative of the maximum number of load flow solutions possible for this network for any type of load specification. For loading conditions close to normal this could be considered as the upper bound. It may be possible to get more solutions than the ZLS for some networks only if we choose small loads at only a few buses.

(6) The proposed algorithm is also useful for determining for a given load profile all the points where the load flow Jacobian has a zero determinant (the points representing steady state stability limit/loadability limit). There are several methods proposed in the literature for finding one of such points corresponding to the normal loading profile. But the investigation here shows that there could be several points (each critical point is one of such point) which satisfy this condition. The proposed algorithm is capable of finding all of them.

(7) One aspect of the proposed method that needs to be investigated further is finding all the ZLS for systems with only PV buses. Since the proposed scheme is dependent on explicit search, this could be quite demanding if the systems have a large number of PV buses. One line of investigation that appears promising is to find as many ZLS as possible using a limited explicit search (say using $2^{N}$ searches) and then trace the solution trajectories for a number of simple and appropriately chosen load profiles. Each such trace could give several other ZLS and continuing this effort (which is not exponentially growing), it may be possible to find all the ZLS for larger systems with only a reasonable amount of computation.

(8) One issue that needs discussion/debate is whether MLFS found using reduced networks could be considered as solutions for the original network, because they are obtained using a subset of nodes after eliminating the isolated nodes of the given network. These solutions indeed satisfy the load flow equations of the given system with the voltages of boundary nodes and isolated nodes considered as zero. However, this appears to be a trivial solution. It is hoped that a consensus can emerge with further investigations.

\section{Conclusion}

A new approach for finding all the (multiple) solutions of load flow equations has been proposed. A very interesting aspect of the proposed scheme is that it is based on linear network analysis and the well-known Newton's method for load flow in rectangular coordinates. The proposed method involves obtaining a sequence of load flow solutions starting from a set of unique points for any system. These starting points are unique to a system and are the zero load solutions of the load flow equations. A scheme of obtaining them efficiently is proposed. The feasibility of the proposed method has been established by obtaining the multiple solutions not only for all systems used by earlier researchers in this area but also for some larger systems not considered by any one so far. Another important feature of the proposed method is that it can not only find the multiple solutions for any given loading condition, but can also find them, corresponding to any multiple (positive or negative) of this load, with no additional effort. In addition this method enables the determination of all the points at which the determinant of load flow jacobian is zero for the given load profile, as a set of critical points. The paper also introduces a new scheme for finding pairs of load flow solutions, corresponding to the same load close to a point where the load flow jacobian is singular. As compared to other existing methods, the proposed method is very easy to implement and computationally very attractive. Moreover, it appears that further investigations could help in simplifying the algorithm further. Further, it is envisaged that the algorithm in its present form itself would help researchers to probe into the relations between MLFS and also, MLFS and operational issues further.

\section{References}

[1] Korsak A J 1972 On the question of uniqueness of stable load-flow solutions. IEEE Trans. Power Apparat. Syst. PAS-91(3): 1093-1100

[2] Johnson B K 1977 Extraneous and false load flow solutions. IEEE Trans. Power Apparat. Syst. 96(2): 524-534 
[3] Baillieul J and Byrnes C I 1982 Geometric critical point analysis of lossless power system models. IEEE Trans. Circ. Syst. 29(11): 724-737

[4] Sauer P W and Pai M A 1990 Power system steady-state and the load-flow jacobian. IEEE Trans. Power Syst. 5(4): 1374-1383

[5] Tamura Y, Mori H and Iwamoto S 1983 Relationship between voltage instability and multiple load flow solutions in electric power systems. IEEE Trans. Power Apparat. Syst. PAS-102(5): 1115-1125

[6] Yorino N, Harada S and Cheng H 1997 A method to approximate a closest loadability limit using multiple load flow solutions. IEEE Trans. Power Syst. 12(1): 424-429

[7] Tamura Y, Iba K and Iwamoto S 1980 A method for finding multiple load-flow solutions for general power systems. Proceedings of IEEE/PES Winter Meeting, New York, paper A-80-043-0

[8] Salam F, Ni L, Guo S and Sun X 1989 Parallel processing for the load flow of power systems: the approach and applications. Proceedings of the 28th IEEE Conference on Decision and Control, vol. 3, pp. 2173-2178

[9] Zhao S, Liu H, Cheng S and Chen D 1993 A new method for calculating power system multiple load flow solutions. IEE 2nd International conference on Advances in Power System Control, Operation and Management, APSCOM-93, pp. 274-278

[10] Ma W and Thorp S 1993 An efficient algorithm to locate all the load flow solutions. IEEE Trans. Power Syst. 8(3): 1077-1083

[11] Zhigang W, Yao Z, Chun T S, Wennan S and Yixin Y 2000 A new method to calculate multiple power flow solutions. Proceedings of the 5th International Conference on Advances in Power System Control, Operation and Management, APSCOM 2000, pp. 491-495, Hong Kong
[12] Iba K, Suzuki H, Egawa M and Watanabe T 1990 A method for finding a pair of multiple load flow solutions in bulk power systems. IEEE Trans. Power Syst. 5(2): 582-591

[13] Iwamoto S and Tamura Y 1981 A load flow calculation method for ill-conditioned power systems. IEEE Trans. Power Apparat. Syst. PAS-100(4): 1736-1743

[14] Overbye T J and Klump R P 1996 Effective calculation of power system low-voltage solutions. IEEE Trans. Power Syst. 11(1): 75-82

[15] Liu C-W, Chang C-S, Jiang J-A and Yeh G-H 2005 Toward a cpflow-based algorithm to compute all the type-1 load-flow solutions in electric power systems. IEEE Trans. Circuits Syst. 52(3): 625-630

[16] Rawal K 2014 Load flow loci enumeration and application to voltage stability of radial system ME Thesis, Department of Electrical Engineering, Indian Institute of Science, Bangalore, India

[17] Kusic G 2008 Computer-aided power systems analysis, CRC Press, Taylor and Francis Group, ISBN 978-1-4200-6106-2

[18] Ajjarapu V and Christy C 1992 The continuation power flow: A tool for steady state voltage stability analysis. IEEE Trans. Power Syst. 7(1): 416-423

[19] Price G B 1984 A generalized circle diagram approach for global analysis of transmission system performance. IEEE Trans. Power Apparat. Syst. PAS-103(10): 2881-2890

[20] Molzahn D K, Lesieutre B C and Chen H 2012 Counter example to a continuation-based algorithm for finding all power flow solutions. IEEE Trans. Power Syst. 28(1): 1-2

[21] Rajathy R 2011 Investigations on power system operation and management in restructured market, Ph.D. thesis, Department of Electrical and Electronics Engineering, Pondicherry University, Pondicherry, India Available: http:// shodhganga.inflibnet.ac.in:8080/jspui/handle/10603/5247 